\title{
Cotyledonoid dissecting leiomyoma of the uterus: A report of four cases and a review of the literature
}

\author{
TIANMIN XU ${ }^{1}$, SHUYING WU ${ }^{1}$, RULIN YANG ${ }^{1}$, LIPING ZHAO $^{1}$, \\ MINGXING SUI $^{1}$, MANHUA CUI ${ }^{1}$ and WEIQIN CHANG ${ }^{2}$ \\ Departments of ${ }^{1}$ Obstetrics and Gynecology and ${ }^{2}$ General Surgery, \\ The Second Hospital of Jilin University, Changchun, Jilin 130041, P.R. China \\ Received March 10, 2015; Accepted February 16, 2016
}

DOI: 10.3892/ol.2016.4314

\begin{abstract}
Cotyledonoid dissecting leiomyoma (CDL), also termed Sternberg tumor, is a variant of uterine leiomyoma that is rarely diagnosed by clinical evaluation. At present, $\sim 43$ cases of CDL have been reported in the literature written in the English language. Due to the distinctive grapelike gross appearance of an exophytic mass resembles placental tissue, CDL is often misdiagnosed clinically as an ovarian tumor or uterine sarcoma. Therefore, an awareness of the features of the disease is important to prevent misdiagnosis and overtreatment. The present study reports 4 cases of CDL of the uterus that were treated at the Second Hospital of Jilin University between January 2009 and December 2011. All 4 patients in the current study presented with a palpable asymptomatic pelvic mass, which was detected during physical examinations, and cancer antigen 125 tumor marker levels that were within the normal range. The exploratory laparotomy of the 4 patients revealed lobulated tumors with a grapelike appearance extending from the lateral uterine wall into the ligament or the adjacent tissues. The frozen section and postoperative pathology were diagnosed as CDL. A total abdominal hysterectomy was performed in the first case of a 55-year-old woman that had been in menopause for 7 years. The patient was well and showed no evidence of disease subsequent to 48 months of follow-up. A total abdominal hysterectomy and right salpingo-oophorectomy were performed in the second case of a 43-year-old woman, who was well and showed no evidence of disease subsequent to 26 months of follow-up. A subtotal abdominal hysterectomy and bilateral salpingectomy were performed in the third case of a 37-year-old woman, who was well and showed no evidence of disease subsequent to 27 months of follow-up. A total abdominal hysterectomy
\end{abstract}

Correspondence to: Professor Weiqin Chang, Department of General Surgery, The Second Hospital of Jilin University, 218 Ziqiang Street, Changchun, Jilin 130041, P.R. China

E-mail: changweiqin55@126.com

Key words: cotyledonoid, dissecting, leiomyoma, leiomyoma of the uterus, Sternberg tumor, grape-like tumor and right-salpingectomy were performed with the removal of a retroperitoneal fibroid extension in the fourth case of a 48-year-old woman, who was well and showed no evidence of disease subsequent to 32 months of follow-up.

\section{Introduction}

Cotyledonoid dissecting leiomyoma (CDL) (1), also termed Sternberg tumor (2), is a rare variant of the uterine leiomyoma. At present, 43 cases of CDL have been reported in the English literature (3). An accurate diagnosis of CDL is challenging prior to surgery, as the distinctive grapelike (4) gross appearance of an exophytic mass resembles placental tissue and is almost always misdiagnosed clinically as an ovarian tumor or uterine sarcoma (5-8). The majority of patients with CDL are diagnosed during an exploratory laparotomy (1,9-11), and a frozen section is recommended to be used for the diagnosis of CDL $(7,9)$. In the literature, to preserve fertility, the recommended treatment is resection of intrauterine tumors by myomectomy and extrauterine tumors by excision $(1,5,12,13)$. In women of postmenopausal stage, total abdominal hysterectomy and bilateral salpingo-oophorectomy are recommended with removal of parametrically extended tumors $(6,14-16)$. No standard treatment for CDL has been identified; however, the prognosis of CDL is favorable. In a previous study, follow-up information was available for 25 of the 41 reported patients $(61 \%)$, none of which experienced tumor recurrence or metastases during the follow-up period (17). The present study reports the cases of 4 patients that were treated at the Second Hospital of Jilin University (Changchun, Jilin, China) between January 2009 and December 2011, in order to improve the current understanding of the disease and to avoid misdiagnosis and overtreatment. The study was approved by the Second Hospital of Jilin Unviersity Ethics Committee (approval no. 2016-006).

\section{Case report}

Case 1. A 55-year-old woman, who had experienced menopause for 7 years, presented to the Second Hospital of Jilin University in January 2009 with a 3-month history of a palpable pelvic mass. A pelvic examination revealed a solid mass, which measured $2 \times 3 \mathrm{~cm}$ in size and was attached to 

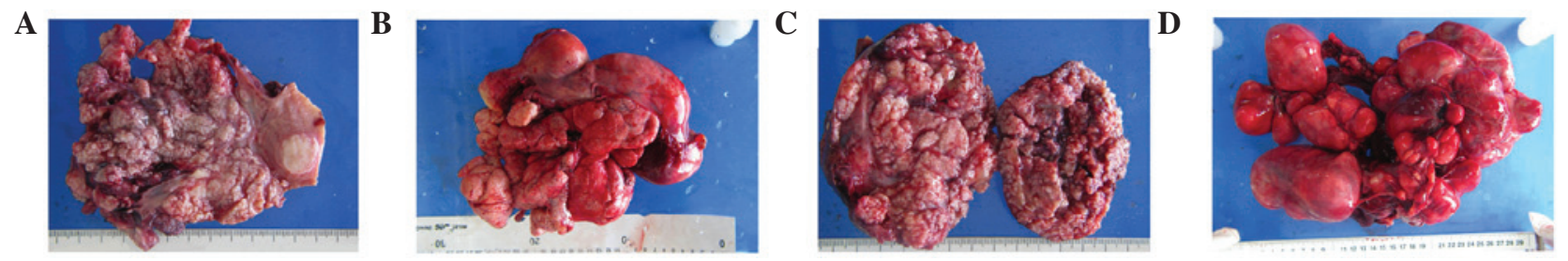

Figure 1. Gross findings for (A) case 1,(B) case 2, (C) case 3 and (D) case 4. (A) Uterus was irregularly enlarged. Multiple myomas merged into a large clustered mass, which extended into the broad ligament. (B) Uterus was slightly enlarged, and the tumor measured $\sim 8 \times 8 \times 7 \mathrm{~cm}$ in size, with multiple heterogeneous myomas in a cluster. The surface of the myomas was smooth. (C) Solid tumor that measured $25 \mathrm{~cm}$ in diameter, with a soft consistency, rich vascularity and smooth surface in the left broad ligament was identified. Multiple coralline-like tumor masses protruded from the surface of the uterus. (D) A lobulated tumor mass with soft consistency was detected extending between the right uterine wall and the surface of the posterior peritoneum.
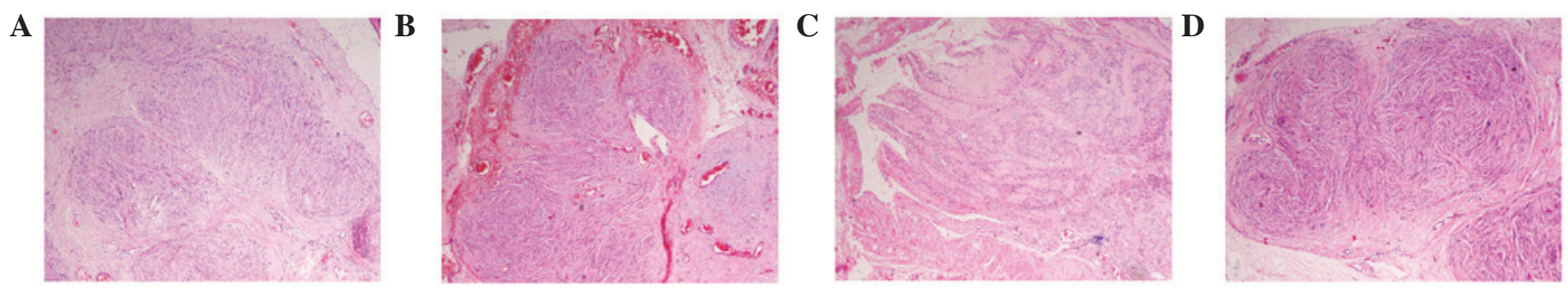

Figure 2. Post-operative pathological section ( $3 \mu \mathrm{m})$ to determine a diagnosis of cotyledonoid dissecting leiomyoma for (A) case 1 , (B) case 2 , (C) case 3 and (D) case 4. (A) The tumor was composed of spindle-shaped smooth muscle cells in a whirlpool pattern. (B) Local interstitial hemorrhage was observed in the tissue. (C) Tumor grew in blood vessels. The vascular wall is located in the lower left corner of the image. (D) Tumor was composed of spindle-shaped smooth muscle cells and local interstitial hemorrhage was observed. Hematoxylin and eosin staining; magnification, x40 (BX-51 Optical Microscope; Olympus Corporation, Tokyo, Japan).

the posterior wall of the uterus, and an irregularly contoured solid mass, which did not result in tenderness and measured $7 \times 5 \mathrm{~cm}$ in size, in the right adnexal region. The transvaginal ultrasound scan demonstrated a regular solid heterogenic low-echo mass that measured $6.9 \times 4.5 \mathrm{~cm}$ in size in the right adnexal region. A blood flow signal was detected around the mass. A low-echo mass measuring $2.3 \times 2.6 \mathrm{~cm}$ was identified protruding from the lower posterior uterine wall. Magnetic resonance imaging (MRI) examination confirmed the presence of an irregular, lobulated, slightly enhanced mass signal that measured $10.0 \times 8.0 \mathrm{~cm}$ in size, lying alongside the right lateral uterine margin. A diagnosis of fibroma ovarii was suggested, and potential serosal myometrial fibroids were not excluded. The cancer antigen 125 (CA125) level was 9 units/ml (normal range, 0-35 units $/ \mathrm{ml}$ ). A diagnosis of ovarian tumor and uterine fibroid was made. The patient underwent an exploratory laparotomy. During the surgery, the uterus was detected to be irregularly enlarged (Fig. 1A). Multiple myomas were identified in the posterior and right wall of the uterus and in the right broad ligament. The diameter of the myomas ranged between 2 and $6 \mathrm{~cm}$. The myomas had merged into a large mass that resembled a cluster of grapes, and extended into the broad ligament. An intraoperative frozen section was obtained, and a diagnosis of CDL of the uterus was made. A total abdominal hysterectomy was performed. The post-operative pathology confirmed that the lesion was CDL (Fig. 2A). The patient was well and showed no evidence of the disease subsequent to 48 months of follow-up.

Case 2. A 43-year-old woman presented to the Second Hospital of Jilin University in November 2009 with a 1-year history of a palpable pelvic mass that had increased in size for 3 months. A pelvic examination revealed a solid fixed mass that measured $\sim 30 \times 20 \times 20 \mathrm{~cm}$ in size. A transvaginal ultrasound scan demonstrated an irregularly contoured heterogenic mass that measured $30 \times 30 \mathrm{~cm}$ in size surrounding the uterus. Blood flow signals were detected inside the mass, and the boundary of the mass was not clear. The CA125 level was 13 units/ml (normal range, 0-35 units $/ \mathrm{ml}$ ). A diagnosis of abdominal-pelvic mass was made. The patient underwent an exploratory laparotomy. During the surgery, a slightly enlarged uterus was observed, measuring $\sim 8 \times 8 \times 7 \mathrm{~cm}$ in size, and multiple heterogeneous myomas were distributed similar to a cluster of grapes around the uterus (Fig. 1B). The surface of the myomas was smooth. An intraoperative frozen section was requested, and a diagnosis of CDL of the uterus was made. A total abdominal hysterectomy and right salpingo-oophorectomy were performed. The post-operative pathology determined that the lesion was CDL with intravascular growth (Fig. 2B). The patient was well and showed no evidence of disease subsequent to 26 months of follow-up.

Case 3. A 37-year-old woman presented to the Second Hospital of Jilin University in May 2010 with a 1-month history of a palpable pelvic mass. A pelvic examination revealed a solid mass that measured $20 \times 10 \times 10 \mathrm{~cm}$ in size. The mass was not easy to move when palpitated, indicating the involvement of surrounding tissues. A transvaginal ultrasound scan demonstrated a mass that measured $30 \times 22 \times 7.1 \mathrm{~cm}$ in size surrounding the uterus, which was not observed separately from the uterus. The echo of the mass resembled the echo of the uterus, and blood flow signals were detected in the mass. The CA125 level was 14 units $/ \mathrm{ml}$ (normal range, 0-35 units $/ \mathrm{ml}$ ). A diagnosis of pelvic mass and uterine fibroid was made. The patient underwent an exploratory laparotomy. During the surgery, a solid tumor that measured $25 \mathrm{~cm}$ in diameter, with a soft 
consistency, rich vascularity and smooth surface was identified in the left broad ligament. Multiple coralline-like tumor masses protruded from the surface of the uterus (Fig. 1C). An intraoperative frozen section was obtained, and a diagnosis of CDL of the uterus was made. A subtotal abdominal hysterectomy and bilateral salpingectomy were performed. The post-operative pathology determined that the lesion was CDL of the uterus (Fig. 2C). The patient was well and showed no evidence of disease subsequent to 27 months of follow-up.

Case 4. A 48-year-old woman presented to the Second Hospital of Jilin University in December 2011 with a 3-month history of fullness felt in the lower abdominal region, and a pelvic mass was detected 1 day prior to admission to the hospital. A pelvic examination revealed a solid mass that measured $14 \times 14 \mathrm{~cm}$ in size attached to the right wall of the uterus. The mass was easy to move when palpitated, therefore the tumor was not adhered to adjacent tissue, and the mass resulted in no tenderness. The transvaginal ultrasound scan revealed an enlarged uterus that measured $11.5 \times 6.7 \times 4.8 \mathrm{~cm}$ in size, and detected numerous echo-poor regions in the wall of the uterus. The largest echo-poor region, measuring $6.7 \times 4.8 \mathrm{~cm}$ in size, was located in the right wall of the uterus. The CA125 level was 15 units/ml (normal range, 0-35 units $/ \mathrm{ml}$ ). A diagnosis of multiple uterine fibroids was made. The patient underwent an exploratory laparotomy. During the surgery, the uterus was $15.0 \times 14.0 \times 10.0 \mathrm{~cm}$ in size (Fig. 1D). A lobulated tumor mass with soft consistency that extended from the right uterine wall to the surface of the posterior peritoneum was detected. The uterine cervix, right fallopian tube, retroperitoneal space, parametrium, posterior leaf of the left broad ligament and the bladder posterior wall were involved. The specimen was excised carefully. Macroscopically, the mass was lobulated and resembled clusters of grapes. An intraoperative frozen section was requested, and a diagnosis of CDL of the uterus was made. A total abdominal hysterectomy and right-salpingectomy were performed, including a removal of a retroperitoneal fibroid extension. The post-operative pathology determined that the lesion of the right broad ligament and the uterus was CDL (Fig. 2D). The patient was well and showed no evidence of disease subsequent to 32 months of follow-up.

\section{Discussion}

CDL was first reported and named by Roth et al (1) in 1966. As the study was dedicated to the late Dr William H. Sternberg (2), who had originally studied the tumor as 'a red seaweed lesion', CDL was also termed the Sternberg tumor. CDL is an atypical leiomyoma with an exophytic appearance and variant growth pattern that was introduced to the WHO Classification of Tumours of Female Reproductive Organs in 2003 (18). CDLs may be present in women of reproductive or postmenopausal age, and CDL has a wide age range of 23-65 years old (mean, 40.3 years) (19). According to the presentation of the intramural dissection or extrauterine extension, CDL is also classified as dissecting leiomyoma, cotyledonoid dissecting leiomyoma or cotyledonoid leiomyoma (1). Due to the lack of typical clinical symptoms, the majority of patients are admitted to hospital with palpable masses and abnormal uterine bleeding. Only a few patients present with no symptoms and identify the disease during physical examinations. Gynecological examinations reveal a palpable pelvic mass that may be detected by ultrasound scan in the uterus corpus or the adnexal region. However, no specific symptoms or tumor markers may be identified in clinical evaluations $(17,20)$. All 4 patients in the present study presented with a pelvic mass that was detected during a physical examination, and demonstrated no evident clinical symptoms or menstruation change, including levels of the CA125 tumor marker that were within the normal range. Ovarian tumor was the most common preoperative diagnosis. Therefore, a lack of specific clinical symptoms and tumor markers contributes to the misdiagnosis of the disease.

Due to the distinctive gross appearance, the diagnosis of the majority of CDLs is challenging prior to surgery. Compared with common uterine fibroids, CDL has its own pathological characteristics (20). Macroscopically, the myometrium of the lateral side of the uterus and the cornua is thinner and weaker compared with the normal myometrium, which enables the tumor to protrude into the pelvic cavity from the uterus. The tumor grows in a dissecting pattern in the intrauterine component, and extends infiltratively from the myometrium into the pelvic cavity $(1,21)$. The extrauterine component exhibits the gross features that resemble cotyledons of the placenta, nodules or clubbed-finger. The cases in the present study also presented with myomas protruding from the lateral uterine myometrium that extended into the broad ligament. In the majority of cases, the extrauterine component of the CDL presents with increased vascularity and attachment to the lateral uterine myometrium or cornua with a narrow fundus or soft fibrous vascular pedicle (22). On electron microscopy, nuclear atypia, mitotic activity and coagulative tumor necrosis are absent (9). The cells are disorganized or swirly in a fascicular or island formation, and exhibit increased vascularity and significant hydropic stroma.

A differential diagnosis is necessary in order to carefully distinguish CDL from other leiomyomas that share similarities with CDL (17). Endometrial stromal sarcoma is a similar leiomyoma where the smooth muscle of the uterus is dissected by the tumor and, occasionally, extends into one lateral ligament. The tumor tissues present with vascular and lymphovascular invasion and a cancerous embolus. Microscopically, deeply stained nuclei, nuclear atypia and mitotic activity may be detected. Intravenous leimyomatosis (IVL) is rarely reported leiomyoma that is characterized by an intravenous component and embolus (23). The typical gross appearance of IVL is multiple nodules protruding into the broad ligament. Occasionally, the tumor mass may also be multinodular and present with an irregular diffusion growth pattern. Mucinous leiomyoma is a leiomyoma that presents with an infiltrative growth pattern, has the appearance of mucinous degeneration and contributes to the empty cysts in various sizes of the mass (24). A degenerated region, which has the appearance of cotton fibres, may be observed at the cut surface, including mucinous cysts filled with transparent mucus. Mucin histochemical staining is conductive to the differential diagnosis of mucinous leiomyoma. Bizarre leiomyoma (4) is a leiomyoma in which the macroscopic features resemble those of conventional leiomyomas. Microscopically, the mass consists of bizarre cells, pleomorphic 
cells, multinucleated giant cell and enlarged hyperchromatic nuclei. Mitotic figures are rarely observed.

Surgery is the best treatment for CDL. However, due to the sarcomatoid operative appearance of the lesion, overtreatment is almost always performed in clinical practice. In 2002, Kim et al (9) first suggested that a frozen section may be obtained during the surgery. In the case that the frozen section indicates a benign tumor, a myomectomy may be considered for young women, particularly for patients that want to preserve fertility. According to the age of the patients, the requirement to bear children and the involvement of the disease, the following surgical methods are always considered, at present $(1,5,6,12-16)$. For older patients that do not express the desire to bear children, a total hysterectomy may be the most appropriate treatment, as demonstrated by the third and fourth cases in the present study. For patients that do not express the desire to bear children, but one of the adnexa is involved, a total hysterectomy and salpingo-oophorectomy is an alternative. For older patients, a total abdominal hysterectomy and bilateral salpingo-oophorectomy may be considered, as demonstrated by the second case in the present study. For older patients of postmenopausal age, a total abdominal hysterectomy and bilateral salpingo-oophorectomy is the best alternative, as demonstrated by the first case in the present study. For patients of a reproductive age that may or may not desire children, a myomectomy is appropriate (3). Saeki et al (3) reported the successful delivery of a baby following a myomectomy for CDL. All patients that were admitted to the Second Hospital of Jilin University underwent exploratory laparotomy. The final surgical method was decided according to the intraoperative findings.

In conclusion, $\mathrm{CDL}$ is a rare disease with a good prognosis. However, the diagnosis of CDL prior to surgery is challenging. Macroscopically, CDL may resemble malignant tumors, which poses a significant challenge for the diagnosis and management of the disease. Microscopically, the determination of a differential diagnosis may be problematic, so the analysis of a frozen section is recommended in order to avoid misdiagnosis and overtreatment. An awareness of leiomyoma variants is required by gynecological surgeons and pathologists for successful differential diagnosis.

\section{Acknowledgements}

The present study was supported by grants from the National Natural Science Foundation of China (grant nos. 81272875 and 81302242), the Jilin Science and Technology Fund (grant nos. 20110755, 20130102094JC and 20140204022YY), the Basic Science Research Fund of Jilin University (grant no. 20142116), the Teaching Reform Project of Jilin University (grant nos. 2013163 and 2014ZH26), Education Science and Planning Issues of Jilin Province (grant no. ZZ1301) and the Doctoral Degree courses of Jilin University (grant no. 2014ZT10).

\section{References}

1. Roth LM, Reed RJ and Sternberg WH: Cotyledonoid dissecting leiomyoma of the uterus. The Sternberg tumor. Am J Surg Pathol 20: 1455-1461, 1996
2. Sternberg WH: Proliferating pelvic angioleiomyomatosis (red seaweed lesion). In: Proceedings of the 9th George Papanicolaou Memorial. Seminar in Gynecologic Pathology, Las Vegas, NV, 1979.

3. Saeki H, Suzuki C, Yamasaki S, Hashizume A, Izumi H, Suzuki F, Ishi K, Nojima M and Hino O: Cotyledonoid dissecting leiomyoma of the uterus: Report of two cases. Arch Gynecol Obstet 291: 357-361, 2015

4. David MP, Homonnai TZ, Deligdish L and Loewenthal M: Grape-like leiomyomas of the uterus. Int Surg 60: 238-239, 1975

5. Tanaka H, Toriyabe K, Senda T, Sakakura Y, Yoshida K, Asakura T, Taniguchi $\mathrm{H}$ and Nagao K: Cotyledonoid dissecting leiomyoma treated by laparoscopic surgery: A case report. Asian J Endosc Surg 6:122-125, 2013

6. Kim NR, Park CY and Cho HY: Cotyledonoid dissecting leiomyoma of the uterus with intravascular luminal growth: A case study. Korean J Pathol 47: 477-480, 2013.

7. Saeed AS, Hanaa B, Faisal AS and Najla AM: Cotyledonoid dissecting leiomyoma of the uterus: A case report of a benign uterine tumor with sarcomalike gross appearance and review of literature. Int J Gynecol Pathol 25: 262-267, 2006.

8. Onu DO, Fiorentino LM and Bunting MW: Cotyledonoid dissecting leiomyoma as a possible cause of chronic lower back pain. BMJ Case Rep 11:2013, 2013.

9. Kim MJ, Park YK and Cho JH: Cotyledonoid dissecting leiomyoma of the uterus: A case report and review of the literature. J Korean Med Sci 17: 840-844, 2002.

10. Driss M, Zhioua F, Doqhri R, Mrad K, Dhouib R and Romdhane KB: Cotyledonoid dissecting leiomyoma of the uterus associated with endosalpingiosis. Arch Gynecol Obstet 280: 1063-1065, 2009.

11. Blake EA, Cheng G, Post MD and Guntupalli S: Cotyledonoid dissecting leiomyoma with adipocytic differentiation: A case report. Gynecol Oncol Rep 11: 7-9, 2014.

12. Chávez Martínez S, Arias González ML, Silva López RM and Villagrán Uribe JA: Cotyledonoid dissecting leiomyoma of the uterus. A malignant-looking benign tumor. Ginecol Obstet Mex 80: 528-533, 2012 (In Spanish).

13. Raga F, Sanz-Cortés M, Casañ EM, Burgues O and Bonilla-Musoles F: Cotyledonoid dissecting leiomyoma of the uterus. Fertil Steril 91: 1269-1270, 2009.

14. Gezginç K, Yazici F, Selimoğlu R and Tavli L: Cotyledonoid dissecting leiomyoma of the uterus with intravascular growth in postmenopausal woman: A case presentation. Int J Clin Oncol 16: 701-704, 2011.

15. Soleymani Majd H, Ismail L, Desai SA and Reginald PW: Epithelioid cotyledonoid dissecting leiomyoma: A case report and review of the literature. Arch Gynecol ObstetArch 283: 771-774, 2011.

16. Weissferdt A, Maheshwari MB and Downey GP: Cotyledonoid dissecting leiomyoma of the uterus: A case report. Diagn Pathol 2: 18, 2007.

17. Smith CC, Gold MA, Wile G and Fadare O: Cotyledonoid dissecting leiomyoma of the uterus: A review of clinical, pathological, and radiological features. Int J Surg Pathol 20: 330-341, 2012.

18. Tavassoli F and Devilee P (eds): World Health Organization Classification of Tumours. Pathology and Genetics of Tumours of the Breacst and Female Genital Organs. IARC Press, Lyon, p241, 2003.

19. Ersöz S, Turgutalp H, Mungan S, Güvend ${ }_{1}$ G and Güven S: Cotyledonoid leiomyoma of uterus: A case report. Turk Patoloji Derg 27: 257-260, 2011.

20. Makharoblidze E, Goishvili N, Mchedlishvili M, Khakhutaishvili I and Jangavadze M: Unusual types of smooth muscle tumors of uterine corpus: Case reports and literature review. Georgian Med News 216: 7-11, 2013.

21. Mathew M, Gowri V, Al Hamdani A, Machado L, Rao K and Shabnam S: Cotyledonoid leiomyoma in pregnancy. Obstet Gynecol 109 (Suppl): 509-511, 2007.

22. Soleymani Majd H, Ismail L, Desai SA and Reginald PW: Epithelioid cotyledonoid dissecting leiomyoma: A case report and review of the literature. Arch Gynecol Obstet 283: 771-774, 2011.

23. Jordan LB, Al-Nafussi A and Beattie G: Cotyledonoid hydropic intravenous leiomyomatosis: A new variant leiomyoma. Histopathology 40: 245-252, 2002.

24. Radojković M, Stojanović M, Gligorijevioć J, Stanojević G, Kovarevios P, Petković TR, Pecić V and Rancić Z: Giant primary retroperitoneal myxoid leiomyoma: A case report. Vojnosanit Pregl 70: 522- 525, 2013. 